\title{
Proceso de enfermería a
}

\section{persona con diagnóstico}

médico de embolia pulmonar e

\section{insuficiencia multiorgánica}

\section{Autores:}

Bryan Martín Gómez González. Licenciado en Enfermería. Especialista en Enfermería en Cuidados Intensivos. Departamento de Enfermeria/Universidad de Sonora. Mail: bryan.gomez@unison.mx

María Olga Quintana Zavala. Licenciada en Enfermería. Doctora en Enfermería. Universidad de Sonora.

Mail: olga.quintana@unison.mx

María Alejandra Favela Ocaño. Licenciada en Enfermería. Maestra en Ciencias en Enfermería. Universidad de Sonora.

Mail: Alejandra.favela@unison.mx

José Iván Nava Luna. Licenciado en Enfermería. Maestro en Educación. Universidad de Sonora.

Mail: jose.nava@unison.mx

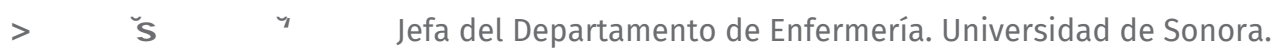

Mail: claudia.figueroa@unison.mx

* Sin conflicto de interés

* Trabajo derivado de tesis de Especialidad de Enfermería en Cuidados Intensivos.

* Financiamiento: sin financiamiento externo

\section{> Resumen}

Introducción: El presente artículo muestra la aplicación del proceso de enfermería a una persona de sexo masculino de 68 años, con una condición poco frecuente que predispone la aparición de complicaciones graves, hospitalizado en una unidad de cuidados intensivos. Objetivo: Evidenciar el cuidado integral de enfermería brindado a un paciente críticamente enfermo con diagnóstico médico de embolia pulmonar e insuficiencia multiorgánica. Desarrollo: Se empleó el proceso de enfermería en todas sus etapas y como guía para la valoración se utilizó los patrones funcionales de salud de Marjory Gordon y para la planifi ación del cuidado de enfermería se manejó el lenguaje estandarizado de la Asociación Norteamericana de diagnósticos de Enfermería, la clasifi ación de resultados e intervenciones de enfermería. Conclusiones: Aún con la aplicación del cuidado organizado por medio del proceso de enfermería, el paciente presenta desenlace fatal, sin embargo, es necesario evidenciar los cuidados de enfermería otorgados, lo que podría ayudar al cuidado de pacientes con respuestas humanas similares y mejoría del pronóstico.

> Palabras claves: Cuidados críticos, embolia pulmonar, insuficiencia multiorgánica, proceso de enfermería, informes de casos (DeCS - BIREME). 


\section{Nursing care for a patient}

with a medical diagnosis of

massive pulmonary embolism

and multiple organ failure

\section{> Abstract}

Introduction: This article shows the application of the nursing process to a 68-year-old male adult, with a rare condition that predisposes the appearance of serious complications, hospitalized in an intensive care unit. Objective: To demonstrate the comprehensive nursing care provided to a critically ill patient with a medical diagnosis of massive pulmonary embolism and multiple organ failure. Development: The nursing process was used in all its stages and as a guide for the assessment, the functional health patterns of Marjory Gordon were used, for the planning of nursing care the standardized language of the North American Association of Nursing diagnoses was used. the classification of nursing outcomes and interventions. Conclusions: Even with the application of organized care through the nursing process, the patient has a fatal outcome, however, it is necessary to show the nursing care provided, which could help the care of patients with similar human responses and improved prognosis.

> Keywords: Intensive care, massive pulmonary thromboembolism, multiorgan failure; nursing process, case reports (DeCS - BIREME). 


\section{, Introducción}

La aplicación del método científi o en el ámbito profesional de enfermería consiste en aplicar el proceso enfermero, lo cual permite realizar cuidados con fundamento en investigaciones científ cas, lógicas y sistemáticas, además, como una primera etapa del proceso enfermero, la valoración de enfermería, es sumamente importante y para ello existen diferentes estructuras recomendadas (1).

Una de las estructuras existentes es la estructura de los patrones funcionales de salud, la cual posee influencia de factores biológicos, culturales, sociales y espirituales; y son una conformación de conductas comunes en los individuos, que c ontribuyen a su s alud y calidad de vida. Junt o con lo anterior, también existen los patrones disfuncionales, los cuales se encuentran en procesos de enfermedad. El juicio de si se tr ata de un patrón funcional o disfuncional se realiza con base en datos de valoración del individuo (2).

Los estudios de c aso son considerados por algunos autores del área de la salud como una clase de diseños, ya sea experimentales o no, cualitativos o cuantitativos, como herramienta para la investigación científi a; a través del caso clínico se mide y registra la conducta del individuo o grupo de individuos involucrados $(3,4)$.

El uso de las taxonomías como son Diagnósticos Enfermeros (NANDA), Clasifi ación de Resultados en Enfermería (NOC) y la Clasifi ación de Intervenciones en E nfermería (NIC), permit en que el profesional realice un mejor desarrollo en la descripción y resolución de los casos clínicos. Estas taxonomías logran la unifi ación del lenguaje enfermero, brindado así herramientas que favorecen la comunicación entre los profesionales de enfermería de los distintos niveles de atención, en este caso, del personal que brinda cuidados intensivos en el quehacer diario del profesional y establece una base de conocimientos científi os $(5,6)$.

El artículo se trata de un caso clínico y proceso de enfermería a paciente hospitalizado en una uni dad de cuidados intensivos de un hospital privado. La estructura de la valoración de enfermería se llevó a cabo sobre la base del marco de valoración de patrones funcionales de salud Marjory Gordon. El propósito principal de este artículo es evidenciar el cuidado integral de enfermería brindado a un paciente críticamente enfermo con diagnóstico médico de embolia pulmonar e insuficiencia multiorgánica, así como, el aspecto emocional, la relación terapéutica y la humanización del cuidado dirigido a una persona gravemente enferma y a su familia.

\section{) Descripción del caso}

Se trata de una per sona de sexo masculino, con 68 años de edad, quien fue dado de alta después de haber sido operado de instrumentación de columna por ant ecedentes de esc oliosis y cif osis. Posteriormente presenta difi ultad respiratoria, fieb e no cuantifi ada y al agravarse los síntomas es trasladado al servicio de urgencias.

Dentro de la valoración de urgencias, se le realiza tomografía de tórax donde se evidencia atelectasia de segmento medial del lóbulo derecho y signos de condensación en asociación, se diagnostica con neumonía adquirida en la comunidad (NAC) y se inicia antibioticoterapia y oxigenoterapia con mascarilla con reservorio, además de ingresar a la Unidad de Cuidados Intensivos (UCI) por presentar $\mathrm{SpO} 2<90 \%$, polipnea (>25 rpm) y taquicardia (frecuencia cardíaca >105 lpm a expensas de fibr lación auricular). Tras su estancia en $\mathrm{UCl}$, mejor a cuadro infeccioso, cursa estable, alerta, cooperador, sin apoyo de aminas, solo requiriendo de apo- 
yo con dispositivo de oxigenoterapia (tipo Bipap) nasal para pernoctar, por lo que días posteriores se programa para reintervención quirúrgica (con el fin de realizar corrección e instrumentación de columna en segundo tiempo quirúrgico); posterior a cirugía regresa al área de $\mathrm{UCl}$, con difi ultad para extubación endotraqueal posoperatoria, $\mathrm{SpO} 2$ $72 \%$, frecuencia cardíaca de $62 \mathrm{lpm}$, tendencia a la hipotensión arterial $(70 / 40 \mathrm{mmHg}$, PAM $51 \mathrm{mmHg}$ ), requiriendo de fármacos vasoactivos (norepinefrina y vasopresina) para mantener presión arterial. En las próximas horas presenta evolución tórpida, se realizan estudios de gabinete y se dia gnostica con embolismo pulmonar ma sivo secundario a uso de material cemento quirúrgico. Días posteriores se reintenta extubación sin éxito, por lo que continua bajo sedoanalgesia (dexmetomidina y fentanilo) con Rass -2 puntos, Campbell 0 .

\section{Antecedentes personales}

- Patológicos: Neuralgia crónica tratada con pregabalina 75 mg/24 h (2016), hiperplasia prostática benigna con tratamiento de tamsulosina $0.4 \mathrm{mg} / 24 \mathrm{~h}$ (2017). Cifosis y escoliosis dorsal (2017), posoperado de instrumentación de columna dorsal (2017), insuficiencia venosa/linfática (2017), fibri ación auricular de respuesta ventricular lenta (2017) tratada con amiodarona $200 \mathrm{mg} / 24 \mathrm{~h}$.

- No patológicos: negados.

- Heredofamiliares: Padre y madre finados por diabetes mellitus 2 , a los 54 y 62 años respectivamente.

\section{> Metodología}

Se aplico el proceso de enfermería en todas sus etapas, ya que se ef ectuó la valoración, diagnósticos, planeación, ejecución y evaluación del cuidado de enfermería. Se realizó la valoración de enfermería según patrones de salud de Marjory Gordon. Aplicando una guía de valoración estructurada para tal fin. Se tomo en cuenta el consentimiento informado de los familiares del paciente para realizar la valoración. El plan de cuidados se realizó de acuerdo con los diagnósticos encontrados y se ejecutaron las intervenciones y su evaluación según lo establecido en el plan de cuidados.

\section{Valoración de enfermería según Patrones Funcio- nales de Salud}

- $\quad$ Patrón de mantenimiento y percepción de la salud: Alcoholismo, tabaquismo, toxicomanías negadas. Sigue indicaciones médicas, hábitos de higiene adecuados; condiciones del hog ar adecuadas.

- Patrón nutricional - metabólico: Disminución de peso en los últimos meses $(10 \mathrm{~kg})$. Nutrición enteral por sonda na soyeyunal $(2886.07 \mathrm{kcal} / 24$ horas), normotérmico, IMC: $24 \mathrm{~kg} / \mathrm{m} 2$. (normal). En piel, presenta fragilidad, pérdida de la turgencia y palidez, extravasación plasmática en miembros torácicos, como abrasiones en cara externa de ambos antebrazos de dos a tres centímetros, además de hemorragia leve. Presenta herida quirúrgica en área toracolumbar con datos de infección, inadecuado afrontamiento, presencia de eritema, secreción purulenta, y perímetro necrótico asociadas a postración, posición y postura corporal, además de difi ultad para cambios de posición en cama. En área lumbosacra y en hélix de oreja derecha lesiones por presión estadío II (según la Agency for Healthcare Research and Qua lity), en mucosa bucofaríngea con lesiones ulcerosas sin sangrado secundarias a intubación orotraqueal, sin ref ejo nauseoso o tusígeno; presencia de sarro en encías y lengua, halitosis. Abdomen globoso, distendido a expensas de visceromegalia y aire, circunferencia abdominal aumentada $(116 \mathrm{~cm})$, asimétrico; a la palpación se encuentra abdomen semi depre- 
sible, presencia de masa abdominal que se localiza en región de hipocondrio y f anco derechos a región umbilical; ruidos peristálticos presentes ( 3 a 4 por minuto), a la percusión se encuentra hipertimpánico. Osmolaridad sérica plasmática elevada (356.01 mOsm/L). Tasa metabólica basal para paciente en estado crítico (Fórmula de Long): $2059.31 \mathrm{kcal} / 24$ horas. Paraclínicos: datos de choque séptico, desequilibrio hidroelectrolítico, estado hipercatabólico [(leucocitosis $(24.4 \mathrm{mil} / \mathrm{mm} 3)$, anemia $(7.6 \mathrm{~g} / \mathrm{dl})$, linfocitemia $(1.94 \%)$, neutróf los (96.1\%), hiperglucemia (114.5 g/dl), hipernatremia (150. $\mathrm{mEq} / \mathrm{l})$, hipokalemia (2.43 mEq/l), hipocalcemia $(7.9 \mathrm{mEq} / \mathrm{l})$, hiperfosfatemia (5.64 mEq/l), elevación de deshidrogenasa láctica (279.2 IU/l), hipoproteinemia ( $4.3 \mathrm{~g} / \mathrm{dl})$, hipoalbuminemia (2.74 $\mathrm{g} / \mathrm{dl}$ ), hipoglobulinemia (1.6 g/dl), hipocloresterolemia $(77.0 \mathrm{mg} / \mathrm{dl})$.

Patrón de eliminación: No pr esentó evacuación intestinal durante la valoración. Región perianal íntegra. Presencia de sonda v esical tipo Foley $16 \mathrm{Fr}$ a derivación, sin datos de infección. Gasto urinario dentro de rango normal $(112 \mathrm{ml} / \mathrm{h}$ [1.3 ml/kg/h]), prepucio edematizado y restricción para retracción, higiene adec uada. Pérdidas insensibles en 24 horas de $924 \mathrm{ml}$. Tasa de f Itración glomerular conservada (91.8 ml/min/1.73m2). Paraclínicos: uremia (194.5 mg/dl), BUN $90.9 \mathrm{mg} / \mathrm{ml}$, creatinina normal $(0.94 \mathrm{mg} / \mathrm{dl})$. Examen general de orina patológico con datos de infección: orina turbia, pH ácido (5.0), hematuria microscópica, bacterias y esterasa leucocitaria positivas.

- $\quad$ Patrón actividad - ejercicio: Presenta escoliosis dorsal y cifosis torácica, expansión torácica asimétrica; vesiculares y broncovesiculares disminuidos, estertores y roncus en ambos hemit órax, hipoventilación en área basal de hemitórax derecho lo cual concuerda con radiografía de tórax (atelectasia del segmento medial del lóbulo dere- cho y signos de c ondensación; hiperdensidad del lóbulo medio del pulmón der echo con signos de condensación y atr apamiento parenquimatoso). Cuenta con apoyo ventilatorio (VMA). P resenta sello de agua en hemitórax derecho con gasto escaso de $10.5 \mathrm{ml} / \mathrm{h}$ hemático. Índice de Kirby (IK): 142 (lesión pulmonar moderada). Presenta f brilación auricular persistente de respuesta ventricular rápida por telemetría, arrítmico; normotenso. Llenado capilar proximal y dist al tardío. Riesgo de caídas según escala Downtown: riesgo de caídas alto (11 puntos). Presenta alcalosis metabólica compensada ( $\mathrm{pH}$ : 7.52, PaO2:76 mmHg, PaCO2: $45 \mathrm{mmHg}, \mathrm{HC}$ 03: $36.7 \mathrm{mmol} / \mathrm{l}$, BE positiv o 12.6 $\mathrm{mmol} / \mathrm{l})$, así como consumo de oxígeno aún normal (Hemodinamia in vasiva por método de Fick: ASC: $2.11 \mathrm{~m} 2, \mathrm{CaO} 2:$ 9.916, VL: $60 \mathrm{ml} / \mathrm{l}, \mathrm{IC}: 1.84$, IS: 0.028, RVS:1299.5 dinas, ITVI: 1.55, DO2: 18.24.)

- $\quad$ Patrón de sueño - desc anso: Bajo sedoanalgesia (dexmetomidina $0.7 \mathrm{mcg} / \mathrm{kg} / \mathrm{min}$ y fentanilo $2.5 \mathrm{mcg} / \mathrm{kg} / \mathrm{h}$ ).

- $\quad$ Patrón cognitivo - perceptivo: Utilización de auxiliares externos visuales (lentes), así como hipoacusia bilateral. RASS -2 (sedación ligera), Campbell 0 (sin dolor). Hiposensibilidad corneal, pupilas isocóricas e hiporef ectivas a estímulo luminoso $(2 \mathrm{~mm})$; presencia de sequedad en ambos ojos.

- Patrón autopercepción y concepto de sí mismo: No valorable.

Patrón rol - relaciones: Jubilado, casado y padre de tres hijos, no trastornos en relaciones intra/interpersonales.

Patrón sexualidad - reproducción: Vida sexual no activa. Sin prácticas de autoexamen mamario ni testicular mensual. Presenta pene ede matizado, difícil visualización de meato, sin datos de infección. Testículos descendidos con hidrocele. Mamas de tipo I, simétricas. 
Patrón de afrontamiento - tolerancia al estrés: No valorable.

- $\quad$ Patrón de valores y creencias: Religión católica, no afecta tratamiento multidisciplinario del equipo de salud.

\section{Plan de cuidados}

El plan de cuidados de enfermería consiste en un conjunto de actuaciones $\mathrm{p}$ ara la detección, descripción, planif cación, tratamiento y evaluación de las respuestas humanas de los pacientes. Se presentan tres de los principales diagnósticos de enfermería detectados, los resultados esperados y las intervenciones de enfermería en el formato de plan de cuidados

\section{Consideraciones éticas}

Protección de personas: Se realiza el caso clínico basado en lo establecido en el artículo 17 del Re- glamento de la Ley General de Salud en Materia de Investigación para la Salud, el cual indica: "se considera la investigación sin riesgo: al ser un estudio que emplea técnicas y métodos de investigación documental retrospectivos y no se realiza ninguna intervención o modif cación intencionada en las variables f siológicas, psicológicas y sociales del individuo que participa en el estudio, entre los que se c onsidera: cuestionarios, revisión de expedientes clínicos y otros, en los que no se l e identif que ni se traten aspectos sensitivos de su conducta" (7).

Conf dencialidad: Según lo establecido en el artículo 14 del Reglamento de la Ley General de Salud en Materia de Investigación para la Salud, el cual señal: "las investigaciones en seres humanos se protegerá la privacidad del individuo sujeto de investigación, identif cándolo solo cuando los resultados lo requieran y éste lo autorice, para lo cual no es necesario el nombre del mismo" (7). 
TABLA 1. DIAGNÓSTICO DE ENFERMERÍA “DETERIORIO INTERCAMBIO DE GASES”

Diagnóstico de Enfermería: 00030 Deterioro del intercambio de gases $\mathrm{r} / \mathrm{c}$ ventilación-perfusión y cambios en la membrana álveo-capilar $\mathrm{m} / \mathrm{p}$ $\mathrm{pH}$ arterial anormal, gasometría arterial anormal (HCO3: $36.7 \mathrm{mmol} / \mathrm{l}$, lactato $1.6 \mathrm{mmol} / \mathrm{l}, \mathrm{pH}: 7.52, \mathrm{BE}: 12.6 \mathrm{mmol} / \mathrm{l}$.)

Def nición: Exceso o déf cit en la oxigenación y/o eliminación de dióxido de carbono en la membrana alvéolo-capilar.

Dominio 03: Eliminación e intercambio

Clase 04: Función respiratoria

Resultado de Enfermería NOC: Estado respiratorio: intercambio gaseoso (0402) (28).

Def nición: Intercambio de $\mathrm{CO} 2$ y 02 para mantener las concentraciones de gases arteriales

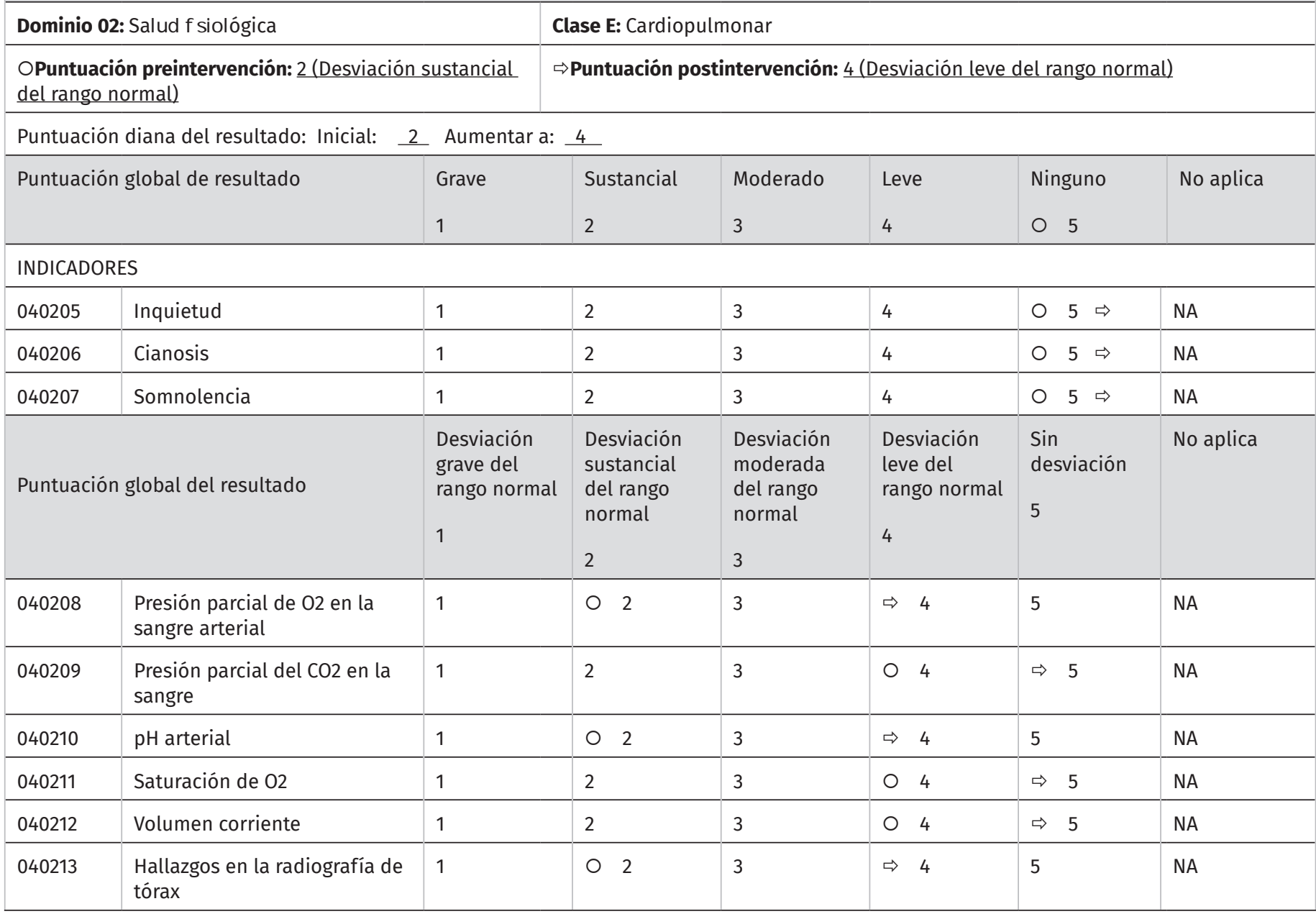




\section{Manejo del equilibrio acidobásico: alcalosis metabólica.}

Def nición: Favorecer el equilibrio acidobásico y prevenir las complicaciones secundarias a unos niveles séricos de HCO3 mayores de lo deseado.

Campo 02: Fisiológico: Complejo

\section{Actividades:}

191201 Mantener la vía aérea permeable.

191202 Monitorizar el patrón respiratorio.

191204 Monitorizar las posibles etiologías antes de intentar tratar los desequilibrios acidobásicos, pues es más ef caz tratar la etiología que el desequilibrio.

191205 Determinar los trastornos que requieren intervención directa frente a los que requieren tratamiento de soporte.

191206 Monitorizar las causas de acumulación de HCO3 o de pérdida de ión hidrógeno (por ejemplo pérdida de líquido gástrico, vómitos, sondaje nasogástrico, diarrea persistente, diuréticos de asa o tiazídicos, f brosis quística, síndrome posthipercapnia, aldosteronismo primario).

191209 Obtener muestras para el análisis de laboratorio del equilibrio acidobásico.

191210 Monitorizar la gasometría arterial y los niveles séricos y urinarios de electrólitos.

191220 Monitorizar las entradas y salidas.

191221 Monitorizar las complicaciones de las correcciones de los desequilibrios acidobásicos (por ejemplo la reducción rápida de la alcalosis metabólica provoca acidosis metabólica).

191225 Evitar la administración de sustancias alcalinas.

191231 Monitorizar las manifestaciones neurológicas y/o neuromusculares de la alcalosis metabólica.
Clase G: Control de electrólitos y acidobásico

Fundamento de la intervención: Cuando existe un predominio de las entradas de agua sobre las pérdidas de la misma, se produce un aumento en el contenido acuoso del organismo. El agua corporal se elimina principalmente por tres vías: La pulmocutánea, la renal y la digestiva. Por la primera vía se realiza la eliminación de agua de manera constante, mientras que la vía renal es variable. En ocasiones no se tiene un adecuado control de los ingresos/egresos y se logra un exceso del volumen, lo que ocasiona complicaciones como HTA, edema, dilución hemodinámica, entre otras, por lo que es importante el control hidroelectrolítico.

Evaluación: La puntuación de la escala diana aumentó de 2 puntos a 4 puntos. Objetivo logrado. 
Diagnóstico de Enfermería: 00100 Retraso en la recuperación quirúrgica r/c cirugía mayor, deterioro de la movilidad, infección del sitio quirúrgico perioperatorio, procedimiento quirúrgico prolongado $\mathrm{m} / \mathrm{p}$ deterioro de la movilidad, evidencia de interrupción de la curación de la herida quirúrgica, tiempo excesivo requerido para la recuperación

Def nición: Aumento del número de días postoperatorio requeridos para iniciar y realizar actividades para el mantenimiento de la vida, la salud y el bienestar (27).

Dominio 11: Salud funcional

Clase D: Autocuidado

Resultado de Enfermería NOC: Recuperación quirúrgica: convalecencia (2304) (28).

Def nición: Grado en que una persona alcanza la función f siológica, psicológica y del rol después del alta desde la unidad de reanimación posquirúrgica hasta la última visita clínica después de la operación.

\begin{tabular}{|c|c|c|c|c|c|c|c|}
\hline \multirow{2}{*}{$\begin{array}{l}\text { Dominio 02: Salud f siológica } \\
\text { OPuntuación preintervención: } 2 \\
\text { (Desviación sustancial del rango } \\
\text { normal) }\end{array}$} & & \multicolumn{6}{|c|}{ Clase AA: Respuesta terapéutica } \\
\hline & & \multicolumn{6}{|c|}{$\Rightarrow$ Puntuación postintervención: $\underline{3}$ (Desviación moderada del rango normal) } \\
\hline \multicolumn{8}{|c|}{ Puntuación diana del resultado: Inicial: $\underline{2}$ Aumentar a: $\underline{4}$} \\
\hline Puntuación global de resultado & $\begin{array}{l}\text { D } \\
\text { g } \\
\text { n }\end{array}$ & $\begin{array}{l}\text { viación } \\
\text { e del rango } \\
\text { mal }\end{array}$ & $\begin{array}{l}\text { Desviación } \\
\text { sustancial del } \\
\text { rango normal } \\
2\end{array}$ & $\begin{array}{l}\text { Desviación } \\
\text { moderada del } \\
\text { rango normal } \\
3\end{array}$ & $\begin{array}{l}\text { Desviación } \\
\text { leve del rango } \\
\text { normal } \\
4\end{array}$ & $\begin{array}{l}\text { Sin desviación } \\
\text { del rango } \\
\text { normal } \\
5\end{array}$ & No aplica \\
\hline
\end{tabular}

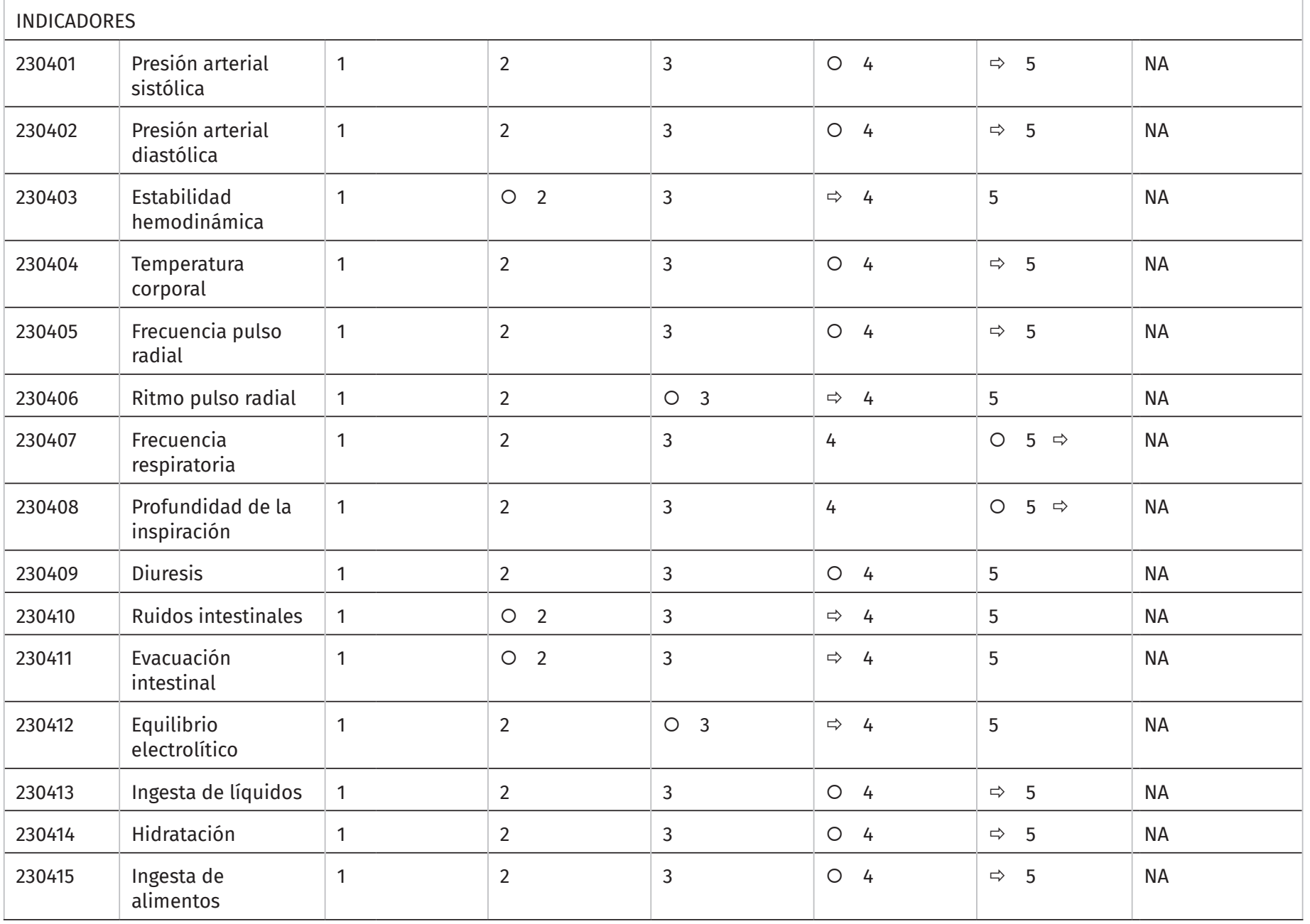




\begin{tabular}{|c|c|c|c|c|c|c|c|}
\hline 230416 & Nivel de glucemia & 1 & 2 & ○ 3 & 4 & $\Rightarrow 5$ & NA \\
\hline 230417 & Integridad tisular & 1 & $\bigcirc 2$ & 3 & $\Rightarrow 4$ & 5 & NA \\
\hline 230418 & $\begin{array}{l}\text { Integridad } \\
\text { neurovascular }\end{array}$ & 1 & ○ 2 & 3 & $\Rightarrow 4$ & 5 & NA \\
\hline 230419 & $\begin{array}{l}\text { Curación de la } \\
\text { herida }\end{array}$ & ○ 1 & 2 & $\Rightarrow 3$ & 4 & 5 & NA \\
\hline 230420 & Ambulación & $\bigcirc 1$ & 2 & $\Rightarrow 3$ & 4 & 5 & NA \\
\hline 230426 & $\begin{array}{l}\text { Adaptación a } \\
\text { cambios corporales } \\
\text { por cirugía }\end{array}$ & 1 & 2 & 3 & 4 & 5 & NA \\
\hline 230428 & $\begin{array}{l}\text { Realización de } \\
\text { actividades de } \\
\text { autocuidado }\end{array}$ & 1 & ○ 2 & 3 & $\Rightarrow 4$ & 5 & NA \\
\hline 230429 & $\begin{array}{l}\text { Reanudación de } \\
\text { las actividades } \\
\text { normales }\end{array}$ & ○ 1 & 2 & 3 & $\Rightarrow 4$ & 5 & NA \\
\hline 230430 & $\begin{array}{l}\text { Reanudación de } \\
\text { la función normal } \\
\text { del rol }\end{array}$ & ○ 1 & 2 & 3 & $\Rightarrow 4$ & 5 & NA \\
\hline \multicolumn{2}{|c|}{ Puntuación global del resultado } & $\begin{array}{l}\text { Grave } \\
1\end{array}$ & $\begin{array}{l}\text { Sustancial } \\
2\end{array}$ & $\begin{array}{l}\text { Moderada } \\
3\end{array}$ & $\begin{array}{l}\text { Leve } \\
4\end{array}$ & $\begin{array}{l}\text { Ninguno } \\
5\end{array}$ & No aplica \\
\hline 230431 & Atelectasia & 1 & ○ 2 & $\Rightarrow 3$ & 4 & 5 & NA \\
\hline 230432 & Neumonía & ○ 1 & 2 & $\Rightarrow 3$ & 4 & 5 & NA \\
\hline 230433 & Dolor & 1 & 2 & 3 & 4 & $\bigcirc 5 \Rightarrow$ & NA \\
\hline 230434 & $\begin{array}{l}\text { Drenado en el } \\
\text { apósito }\end{array}$ & ○ 1 & 2 & $\Rightarrow 3$ & 4 & 5 & NA \\
\hline 230435 & $\begin{array}{l}\text { Drenado del } \\
\text { drenaje }\end{array}$ & ○ 1 & 2 & $\Rightarrow 3$ & 4 & 5 & NA \\
\hline 230436 & $\begin{array}{l}\text { Infección de la } \\
\text { herida }\end{array}$ & ○ 1 & 2 & $\Rightarrow 3$ & 4 & 5 & NA \\
\hline 230437 & $\begin{array}{l}\text { Dehiscencia de la } \\
\text { herida }\end{array}$ & 1 & 2 & 3 & $04 \Rightarrow$ & 5 & NA \\
\hline 230438 & Trombof ebitis & 1 & 2 & 3 & $04 \Rightarrow$ & 5 & NA \\
\hline 230439 & $\begin{array}{l}\text { Trombosis } \\
\text { pulmonar }\end{array}$ & 1 & 2 & O 3 & $\Rightarrow 4$ & 5 & NA \\
\hline 230442 & íleo paralítico & 1 & 2 & 3 & $04 \Rightarrow$ & 5 & NA \\
\hline 230443 & Estreñimiento & 1 & O 2 & $\Rightarrow 3$ & 4 & 5 & NA \\
\hline 230444 & Fatiga & 1 & $\circ 2$ & $\Rightarrow 3$ & 4 & 5 & NA \\
\hline 230445 & Ansiedad & 1 & $\bigcirc 2$ & $\Rightarrow 3$ & 4 & 5 & NA \\
\hline
\end{tabular}


TABLA 4 : INTERVENCIONES DE DIAGNÓSTICO DE ENFERMERÍA “RETRASO EN LA RECUPERACIÓN QUIRÚRGICA"

3664 Cuidado de las heridas: ausencia de cicatrización (29).

Def nición: Guidados paliativos y prevención de las complicaciones de una herida maligna o de otro tipo cuando no es previsible la cicatrización

Campo: 02 Fisiológico: Complejo

Clase: L Control de la piel/heridas

Actividades:

366403 Humedecer los vendajes con solución salina antes de retirarlos

366404 Describir las características de la úlcera, observando el tamaño ubicación, exudado, color, hemorragia, dolor, olor y edema

366405 Registrar los cambios observados en la evolución de la úlcera

Fundamento de la intervención: La cicatrización ocurre de manera directa por epitelización y reparación del tejido en heridas, o bien, la que se realiza en forma aséptica con la unión de los bordes de la herida con puntos de sutura y el tejido de granulación no visible y cicatriz mínima. La herida requiere de una higiene adecuada para que el organismo acelere el proceso de cicatrización y evitar complicaciones.

366406 Observar los signos y síntomas de infección de la herida

366409 Evitar la fricción con gasa durante la limpieza

3664013 Evitar la debridación química o mecánica del tejido

366414 Aplicar medicación tópica (citostáticos, antibióticos, analgésicos)

366416 Utilizar apósitos muy absorbentes cuando haya abundante secreción

366420 Determinar el impacto que la úlcera está teniendo en la calidad de vida del paciente (sueño, apetito, actividad, estado de ánimo, relaciones)

366425 Enseñar los métodos para proteger la herida de golpes, presión y fricción (uso de almohadas, cojines, almohadillas)

Evaluación: La puntuación de la escala diana aumentó de 2 puntos a 3 puntos. Objetivo medianamente logrado. 
TABLA 5: DIAGNÓSTICO DE ENFERMERÍA “EXCESO DE VOLUMEN”

Diagnóstico de Enfermería: 00026 Exceso del volumen de líquidos r/c exceso de aporte de líquidos m/p edema (miembros torácicos, escroto), sonidos respiratorios ocasionales (estertores bibasales).

Def nición: Aumento de la retención de líquidos isotónicos (27).

Dominio 02: Nutrición

Clase 05: Hidratación

Resultado de Enfermería NOC: Equilibrio hídrico (0601) (28).

Def nición: Equilibrio de agua en los compartimientos intracelulares y extracelulares del organismo

Dominio 02: Salud f siológica

Clase G: Líquidos y electrólitos

Puntuación preintervención: $\underline{2}$ (Sustancialmente Puntuación postintervención: 4 (Levemente comprometido/leve) comprometido/grave)

Puntuación diana del resultado: Inicial: $\quad 2$ Aumentar a: 4

\begin{tabular}{|c|c|c|c|c|c|c|}
\hline Puntuación global de resultado & $\begin{array}{l}\text { Gravemente } \\
\text { comprometido } \\
1\end{array}$ & $\begin{array}{l}\text { Sustancialmente } \\
\text { comprometido } \\
2\end{array}$ & $\begin{array}{l}\text { Moderadamente } \\
\text { comprometido } \\
3\end{array}$ & $\begin{array}{l}\text { Levemente } \\
\text { comprometido } \\
4\end{array}$ & $\begin{array}{l}\text { Sin compromiso } \\
5\end{array}$ & No aplica \\
\hline
\end{tabular}

INDICADORES

\begin{tabular}{|c|c|c|c|c|c|c|c|}
\hline 060101 & Presión arterial & 1 & 2 & 3 & 4 & 5 & NA \\
\hline 060102 & $\begin{array}{l}\text { Presión arterial } \\
\text { media }\end{array}$ & 1 & 2 & 3 & 4 & 5 & NA \\
\hline 060103 & $\begin{array}{l}\text { Presión venosa } \\
\text { central }\end{array}$ & 1 & 2 & 3 & 4 & & NA \\
\hline 060105 & Pulsos periféricos & 1 & 2 & 3 & 4 & 5 & NA \\
\hline 060107 & $\begin{array}{l}\text { Entradas y salidas } \\
\text { diarias equilibradas }\end{array}$ & 1 & 2 & 3 & 4 & 5 & NA \\
\hline 060109 & $\begin{array}{l}\text { Peso corporal } \\
\text { estable }\end{array}$ & 1 & 2 & 3 & 4 & 5 & NA \\
\hline 060116 & Hidratación cutánea & 1 & 2 & 3 & 4 & 5 & NA \\
\hline 060117 & $\begin{array}{l}\text { Humedad de } \\
\text { membranas } \\
\text { mucosas }\end{array}$ & 1 & 2 & 3 & 4 & 5 & NA \\
\hline 060118 & Electrólitos séricos & 1 & 2 & 3 & 4 & 5 & NA \\
\hline 060119 & Hematocrito & 1 & 2 & 3 & 4 & 5 & NA \\
\hline 060122 & $\begin{array}{l}\text { Velocidad de pulso } \\
\text { radial }\end{array}$ & 1 & 2 & 3 & 4 & 5 & NA \\
\hline \multicolumn{2}{|c|}{ Puntuación global del resultado: 2} & $\begin{array}{l}\text { Grave } \\
1\end{array}$ & $\begin{array}{l}\text { Sustancial } \\
2\end{array}$ & $\begin{array}{l}\text { Moderado } \\
3\end{array}$ & $\begin{array}{l}\text { Leve } \\
4\end{array}$ & $\begin{array}{l}\text { Ninguno } \\
5\end{array}$ & No aplica \\
\hline 060106 & $\begin{array}{l}\text { Hipotensión } \\
\text { ortostática }\end{array}$ & 1 & 2 & $\Rightarrow 3$ & 4 & 5 & NA \\
\hline 060108 & $\begin{array}{l}\text { Ruidos respiratorios } \\
\text { patológicos }\end{array}$ & 1 & $\bigcirc 2$ & 3 & 4 & 5 & NA \\
\hline 060110 & Ascitis & 1 & 2 & 3 & 4 & $\bigcirc 5 \Rightarrow$ & NA \\
\hline 060111 & $\begin{array}{l}\text { Distensión de las } \\
\text { venas del cuello }\end{array}$ & 1 & 2 & 3 & 4 & $\bigcirc 5 \Rightarrow$ & NA \\
\hline 060112 & Edema periférico & O & 2 & 3 & 4 & 5 & NA \\
\hline
\end{tabular}


TABLA 6 : INTERVENCIONES DE DIAGNÓSTICO DE ENFERMERÍA “EXCESO DE VOLUMEN”

4120 Manejo de líquidos (29).

Def nición: Mantener el equilibrio de líquidos y prevenir las complicaciones derivadas de los niveles de líquidos anormales.

Campo 02: Fisiológico: Complejo

Actividades:

412001 Pesar a diario y controlar la evolución

412003 Realizar un registro preciso de entradas y salidas

412004 Realizar sondaje vesical

412005 Vigilar el estado de hidratación

412006 Controlar resultados de laboratorio relevantes en la retención e líquidos (aumento de la gravedad específ ca, aumento del BUN,

disminución del hematocrito y aumento de la osmolalidad urinaria)

412007 Monitorizar el estado hemodinámico

412008 Monitorizar los signos vitales

412009 Observar si hay indicios de sobrecarga/retención de líquidos

412011 Evaluar la ubicación y extensión del edema

412014 Monitorizar el estado nutricional

412015 Administrar los diuréticos prescritos

412014 Distribuir la ingesta de líquidos en 24 horas

412026 Consultar con el médico, si los signos y síntomas de exceso de volumen de líquidos persisten o empeoran
Clase N: Control de la perfusión tisular

Fundamento de la intervención: Cuando existe un predominio de las entradas de agua sobre las pérdidas de la misma, se produce un aumento en el contenido acuoso del organismo. El agua corporal se elimina principalmente por tres vías: La pulmocutánea, la renal y la digestiva. En ocasiones no se tiene un adecuado control de los ingresos/egresos y se logra un exceso del volumen, lo que ocasiona complicaciones como HTA, edema, dilución hemodinámica, entre otras, por lo que es importante el control hidroelectrolítico.

Evaluación: La puntuación de la escala diana aumentó de 2 puntos a 4 puntos. Objetivo logrado. 
TABLA 7 : DIAGNÓSTICO DE ENFERMERÍA “DETERIORO INTEGRIDAD CUTÁNEA”

Diagnóstico de Enfermeria: 00046 Deterioro de la integridad cutánea r/c alteración en el volumen de líquidos, alteración de la pigmentación, alteración de la sensibilidad, alteración de la turgencia de la piel, deterioro de la circulación, nutrición inadecuada, presión sobre prominencias óseas, alteración en el metabolismo m/p alteración de la integridad de cutánea (UPP de segundo grado en región sacro-dorsal, laceraciones en miembros pélvicos).

Def nición: Alteración de la epidermis y/o de la dermis.

Dominio 11: Seguridad/Protección

Clase 02: Lesión física

Resultado de Enfermería NOC: Integridad tisular: piel y membranas mucosas (1101)

Def nición: Indemnidad estructural y función f siológica normal de la piel y las membranas mucosas.

Dominio 02: Salud f siológica

Puntuación preintervención: 2 (Sustancialmente comprometido/Sustancial)

Clase L: Integridad tisular

Puntuación diana del resultado: Inicial: $\quad 2 \quad$ Aumentar a: 4

\begin{tabular}{|c|c|c|c|c|c|c|}
\hline Puntuación global de resultado & $\begin{array}{l}\text { Gravemente } \\
\text { comprometido } \\
1\end{array}$ & $\begin{array}{l}\text { Sustancialmente } \\
\text { comprometido } \\
2\end{array}$ & $\begin{array}{l}\text { Moderadamente } \\
\text { comprometido } \\
3\end{array}$ & $\begin{array}{l}\text { Levemente } \\
\text { comprometido } \\
4\end{array}$ & $\begin{array}{l}\text { Sin } \\
\text { compromiso } \\
5\end{array}$ & No aplica \\
\hline
\end{tabular}

INDICADORES

\begin{tabular}{|c|c|c|c|c|c|c|c|}
\hline 110101 & Temperatura de la piel & 1 & 2 & 3 & 4 & $05 \Rightarrow$ & NA \\
\hline 110102 & Sensibilidad & 1 & 2 & 3 & 4 & 5 & NA \\
\hline 110103 & Elasticidad & 1 & $\circ 2$ & $\Rightarrow 3$ & 4 & 5 & NA \\
\hline 110104 & Hidratación & 1 & 02 & $\Rightarrow 3$ & 4 & 5 & NA \\
\hline 110106 & Transpiración & 1 & 2 & 3 & 4 & ○ $5 \Rightarrow$ & NA \\
\hline 110108 & Textura & 1 & 02 & $\Rightarrow 3$ & 4 & 5 & NA \\
\hline 110109 & Grosor & 1 & 02 & $\Rightarrow 3$ & 4 & 5 & NA \\
\hline 110111 & Perfusión tisular & 1 & ○ 2 & $\Rightarrow 3$ & 4 & 5 & NA \\
\hline 110112 & $\begin{array}{l}\text { Crecimiento del vello } \\
\text { cutáneo }\end{array}$ & 1 & 2 & 3 & 4 & $\bigcirc 5 \Rightarrow$ & NA \\
\hline 110113 & Integridad de la piel & 1 & $\bigcirc 2$ & $\Rightarrow 3$ & 4 & 5 & NA \\
\hline \multicolumn{2}{|c|}{ Puntuación global del resultado } & $\begin{array}{l}\text { Grave } \\
1\end{array}$ & $\begin{array}{l}\text { Sustancial } \\
2\end{array}$ & $\begin{array}{l}\text { Moderado } \\
3\end{array}$ & $\begin{array}{l}\text { Leve } \\
4\end{array}$ & $\begin{array}{l}\text { Ninguno } \\
5\end{array}$ & No aplica \\
\hline 110105 & Pigmentación anormal & 1 & 02 & $\Rightarrow 3$ & 4 & 5 & NA \\
\hline 110115 & Lesiones cutáneas & 1 & $\bigcirc 2$ & $\Rightarrow 3$ & 4 & 5 & NA \\
\hline 110116 & Lesiones de la mucosa & 1 & 2 & 3 & 4 & $05 \Rightarrow$ & NA \\
\hline 110117 & Tejido cicatricial & 1 & 02 & $\Rightarrow 3$ & 4 & 5 & NA \\
\hline 110118 & Cánceres cutáneos & 1 & 2 & 3 & 4 & $\bigcirc 5 \Rightarrow$ & NA \\
\hline 110119 & Descamación cutánea & 1 & $\bigcirc 2$ & $\Rightarrow 3$ & 4 & 5 & NA \\
\hline 110120 & Raspado cutáneo & 1 & 02 & $\Rightarrow 3$ & 4 & 5 & NA \\
\hline 110121 & Eritema & 1 & $\circ 2$ & $\Rightarrow 3$ & 4 & 5 & NA \\
\hline 110122 & Palidez & 1 & $\circ 2$ & $\Rightarrow 3$ & 4 & 5 & NA \\
\hline 110123 & Necrosis & 1 & 02 & $\Rightarrow 3$ & 4 & 5 & NA \\
\hline 110124 & Induración & 1 & 02 & $\Rightarrow 3$ & 4 & 5 & NA \\
\hline 110125 & Abrasión corneal & 1 & 2 & 3 & 4 & ○ $5 \Rightarrow$ & NA \\
\hline
\end{tabular}


0740 Cuidados del paciente encamado (29).

Def nición: Fomento de la comunidad y la seguridad, así como prevención de complicaciones en el paciente que no puede levantarse de la cama.

Campo 01: Fisiológico: Básico

\section{Actividades:}

74002 Colocar al paciente sobre la cama o colchón terapéutico adecuado

74004 Evitar utilizar ropa de cama con texturas ásperas

74005 Mantener la ropa de cama limpia, seca y sin arrugas

74006 Colocar en la cama una base de apoyo para los pies

74008 Aplicar dispositivos que eviten los pies equinos

74010 Cambiar de posición al paciente, según lo indique el estado de la piel

74015 Girar al paciente inmovilizado al menos cada 2 horas

74016 Vigilar el estado de la piel

74017 Realizar ejercicios de rango de movimiento pasivos y/o activos

74020 Ayudar con las medidas de higiene (uso de desodorante o perfume).

74022 Aplicar medidas prof lácticas antiembólicas.

74026 Monitorizar la aparición de complicaciones del reposo en cama (por ejemplo: hipotonía muscular, dolor de espalda, estreñimiento, aumento del estrés, depresión, confusión, cambios del ciclo de seño, infecciones del tracto urinario, dif cultad en la micción, neumonía)
Clase C: Control de la inmovilidad

Fundamento de la intervención: La inmovilidad y el reposo en cama prolongado, así como la escasa o nula actividad física condicionan la aparición de complicaciones en el organismo, principalmente en los sistemas: cardiovascular, respiratorio, digestivo, musculoesquelético y tegumentario, lo cual predisponen a estancias hospitalarias prolongadas y a la presencia de otras complicaciones.

La movilización en cama precozmente ha demostrado la disminución de aparición de complicaciones, menos días de estancia hospitalaria. La prevención de úlceras por decúbito al realizar cambios posturales del usuario encamado, así como cabecera no menor de $30^{\circ}$, ropa de cama limpia, seca, adecuada nutrición, prof laxis antiembólica, ejercicios favorecen la recuperación de los usuarios.

Evaluación: La puntuación de la escala diana aumentó de 2 puntos a 3 puntos. Objetivo medianamente logrado. 


\section{> Discusión y conclusiones}

Las complicaciones postquirúrgicas más comunes en adultos mayores son daño pulmonar, neumonía, ventilación mecánica prolongada, atelectasias, neumotórax, tromboembolismo pulmonar, entre otros (11-16).

El usuario al que se b asa este caso clínico inició su padecimiento posterior a ser reintervenido quirúrgicamente de instrumentación de columna (corrección de escoliosis y cifosis); pasó de la sala de quirófano a la unidad de cuidados intensivos, por presentar hipotensión arterial severa, síndrome de distrés respiratorio, siendo la causa embolización de material (cemento quirúrgico) utilizado en la cirugía.

Durante su estancia en la unidad de cuidados intensivos presentó evolución tórpida e insuf ciencia orgánica, lo que concuerda con varios autores; los usuarios de edad avanzada (>60 años), con comorbilidades, reintervenciones quirúrgicas, y que son sometidos a cirugía ma yor (tiempo >2 horas), períodos prolongados de uso de fármacos anestésicos y vasoactivos, conllevan una estancia larga en la unidad de cuidados intensivos, así como otras complicaciones (14-21).

A diferencia de la usuaria femenina de 59 años del caso clínico realizado por Vargas, Agudelo y Rozo, y usuaria de 85 años del c aso clínico elaborado por Fornell, Santana y Rionda donde en ambos se realizó la cirugía de vertebroplastía mediante utilización de cemento quirúrgico óseo (metilemetacrilato) presentaron tromboembolismo pulmonar. Las dos situaciones fuer on tratadas de la misma manera que el usuario de este caso clínico (fármacos anticoagulantes, uso de medias compresivas y vigilancia), sin embargo, el usuario presentó otras complicaciones (22-23).
El tiempo promedio de estancia en una unidad de cuidados intensivos es de siet e días, por lo que una estancia mayor a est e tiempo se c onsidera prolongada. La valoración de enf ermería a través de los patrones funcionales permitió identif car las necesidades de atención prioritarias en el usuario, las intervenciones de enfermería plasmadas en los planes de cuidados fueron clave para mantener los indicadores de resultados y paliar algunas situaciones, no obst ante, las complicaciones multiorgánicas continuaron presentándose y condujeron a un desenlace fatal. Aun con las complicaciones descritas, el cuidado del usuario se enfocó a mantener su confort y manejar, de manera paliativa, la sintomatología que fue presentando, además de brindar en todo momento un cuidado humanizado para el usuario y su familia.

El personal de enf ermería que se enc uentra en las unidades de cuidados intensivos, así como el resto del equipo multidisciplinario debe recibir capacitación y documentar estos sucesos, los pacientes que se someten a estos procedimientos o reintervenciones requieren monitorización hemodinámica constante y atención estricta en signos y síntomas indicativos de embolismo pulmonar (17,24-26).

El usuario en est ado crítico requiere de hospitalización en una unidad de cuidados intensivos, donde el enfermero que labora en esta área debe contar con un perf I de actualización permanente, acorde a la complejidad del cuidado que brinda y a los avances existentes, amplio conocimiento técnico-científ co, clínico, acompañado de iniciativa, creatividad, habilidad, destreza, liderazgo, motivación, equilibrio emocional, responsabilidad y ética.

La enfermera o el enf ermero especialista en cuidados intensivos, debe reconocer las emergencias que se presentan y actuar inmediatamente toman- 
do decisiones en coordinación con los profesionales del equipo multidisciplinario de salud, además de ser competente para brindar un c uidado de enfermería individualizado, basado en modelos y teorías, interpretar resultados de laboratorio, leer y reconocer alteraciones cardiacas, conocer parámetros ventilatorios, operar respiradores mecánicos, actuar en reanimación cardiopulmonar, participar en procedimientos, además de establecer una relación terapéutica interpersonal con los pacientes y sus familias, para lo cual se requiere un amplio bagaje de conocimientos teóricos, acompañado de actitudes de solidaridad, respeto, empatía, deferencia, etcétera, así como documentar e investigar temáticas relacionadas con su área de experiencia que promueven las mejores prácticas de cuidado intensivo de enfermería.

Es imprescindible que el profesional de enfermería lleve a cabo estudios de c aso basados en la metodología de enfermería, donde se documenten y posteriormente publiquen, para con ello desarrollar literatura de futuras investigaciones, mejorando así la praxis del gremio y la atención del usuario en estado crítico.

\section{> Referencias}

1. Gordon M. Manual de diagnósticos enfermeros. 10 ed. EUA: Elsevier; 2007. 625 p.

2. Rivera V, Pastoriza P, Díaz C, Huapaya H. Revisión crítica de la taxonomía enfermera NANDA Internacional 2009-2011. Revisión Clínica. Rev Científ ca Enf RECIEN. [Internet]. 2013[Acceso 08 de diciembre 2018];6(1)1-18. Disponible en: http://www. recien.scele.org/documentos/num_6_may_2013/ revis_critica_taxonomia_enfermera.pdf

3. Hernández-Sampieri R, Fernández C, Baptista P. Metodología de la Investigación. 5á ed. México: McGraw-Hill interamericana Editores; 2010. 600 p.
4. Tirado G, Hueso C, Fernández-Gallego MC, Montoya R, Bonill C, Schmidt J. C ómo escribir un caso clínico en Enfermería utilizando Taxonomía NANDA, NOC, NIC. Inde x Enferm [Internet]. 2011. [Acceso 06 diciembre 2018];20(1):111-15. Disponible en: http://scielo.isciii.es/scielo.php?script=sci_art text\&pid=S113212962011000100023

5. Álvarez JL, Castillo F, Fernández D, Muñoz M. Manual de Valoración de Patrones Funcionales Servicio de Salud del Principado de Austrias. [Internet]. 2010. [Acceso 08 diciembre 2018]. Disponible en: https://www.seapaonline.org/UserFiles/ File/Ayuda\%20en\%20consulta/MANUAL\%20VALORACION\%20NOV\%202010.pdf

6. Berman A., Snyder S. Fundamentos de Enfermería. Conceptos, proceso y práctica. España: Pearson Educación. 2013.

7. Reglamento de l a Ley General de Sa lud en Materia de In vestigación para la Salud. México: Diario Of cial de la Federación. [Internet]; 2014. [Acceso 10 febrero 2020]. Disponible en: http:// www.diputados.gob.mx/LeyesBiblio/regley/Reg_ LGS_MIS.pdf

8. Herdman, T.H. NANDA Internacional. Diagnósticos Enfermeros. Def niciones y clasif cación. 2015-2017. España: Elsevier; 2012. 483 p.

9. Moorhead S., Jhonson M., Ma ss M., Swanson E. Clasif cación de resultados de enfermaría (NOC): Medición de Resultados en salud. 5ta. ed. España: 2013. 735 p.

10. Bulechek G., But cher H., Docht erman J., Wagner C. Clasif cación de intervenciones de enfermería (NIC). 6t a. ed. E spaña: Elsevier Mosby; 2013. 976 p.

11. Martos FD, Guzmán BI, González I. Complicaciones posoperatorias en cirugía mayor torácica y abdominal: def niciones, epidemiologia y gra- 
vedad. Revista cubana de cirugía. [Int ernet]. 2016 [Acceso 08 diciembre 2018];55(1):1-15. Disponible en: http://www.revcirugia.sld.cu/index.php/cir/ article/view/274/165

12. Canet J, Gallart L. Predicción de complicaciones postoperatorias pulmonares en una c ohorte basada en una pobl ación quirúrgica. Revista Chilena de Anest esia [Acceso 08 diciembre 2018];48(4):314-323. Disponible en: https:/ /doi. org/10.25237/revchilanestv48n04.06

13. Soares S, Bertoldi L, Da Silva M, Colombini T. Pulmonary function and ph ysical performance outcomes with preoperative physical therapy in upper abdominal surgery: a randomized controlled trial. Clin Rehabil. 2013. [Acceso $08 \mathrm{di}$ ciembre 2018].Jul;27(7):616-27. Disponible en: DOI: $10.1177 / 0269215512471063$

14. Hernández A, Ada H, Mitjans C, Manrique A, Brown J, Sardiñas O. Complicaciones pulmonares posoperatorias en pacientes ingresados en Unidad de Cuidados Intensivos. Rev. Cubana Cir. [Internet]. 2015 [Acceso 08 diciembre 2018];54(3):23545. Disponible en: https://www.medigraphic.com/pdfs/ cubcir/rcc-2015/rcc153e.pdf

15. Bajwa SJS, Kulshrestha A. Diagnosis, Prevention and Management of Postoperative Pulmonary Edema. Ann Med Health Sci Res. 2012;2(2):1805.

16. Blum JM, St entz MJ, Dechert R, Jewell E, Engoren $M$, Rosenberg AL, et a l. Preoperative and Intraoperative Predictors of $\mathrm{P}$ ostoperative Acute Respiratory Distress Syndrome in a General Surgical Population. Anesthesiology. 2013;118:199.

17. Gómez-Sánchez G, Zarazía M., García E. Alto riesgo perioperatorio: riesgo respiratorio, ventilación mecánica y complicaciones postoperatorias respiratorias. Rev. Mex. Anestesiología. [Internet]
2016. [Acceso 08 diciembre 2018];39(1):S79S81. Disponible en: http:/ /www.medigraphic.com/pdfs/ rma/cma-2016/cmas161y.pdf

18. Carrillo-Esper R, de la Torre-León T, Carrillo-Córdova CA, Carrillo-Córdova DM. Actualidades en la def nición, f siopatología y tratamiento de la lesión pulmonar a guda. Rev. Int. Mex. [Internet]. 2015. [Acceso 13 octubre 2018]; 31:57-59, Disponible en: http:/ /www.medigraphic.com/pdfs/medintmex/mim2015/mim155k.pdf

19. Güldner A, Kiss T, Serpa Neto A, Hemmes SN, Canet J, Spieth P M, et a l. Intraoperative Protective Mechanical Ventilation for Prevention of Postoperative Pulmonary Complications. Anesthesiology [Internet]. 2015 [Acceso 08 diciembr e 2018];123:692-713. Disponible en: DOI: 10. 1097/ ALN.0000000000000754.

20. Gordo F., Enciso V. Síndrome de distrés respiratorio agudo, ventilación mecánica y función ventricular derecha. Rev. Med. Int. Medicina Intensiva. Elsevier. [Internet]. 2011. [Acceso 13 octubre 2018];36(2):138-142, Disponible en: http:/ /scielo. isciii.es/pdf/medinte/v36n2/punto.pdf

21. West, J.B. Fisiología y f siopatología pulmonar: estudio de c asos. 2a ed. Bar celona: Wolters Kluwer Health España; 2008. 192 p.

22. Vargas LJ, Agudelo MB, Rozo EJ. Embolia pulmonar por metilmetacrilato. Rev. Col. Card. [Internet] 2018. [Acceso 08 diciembre 2018];25(5):341. Disponible en: https:/ /doi.org/10.1016/j.rccar.2017.08.015

23. Fornell R, Santana JM, Junquera P. Embolismo pulmonar múltiple por cemento acrílico tras vertrobroplastía. Arch Bronconeumol. [Internet] 2010. [Acceso 10 diciembre 2018];46(9):492-97. Disponible en: https:/ / www.archbronconeumol.org/ es-pdf-S0300289610001328 
24. Langona L., Barbosa M., Pinto M. Actuación de la enfermera en la unidad de terapia intensiva neonatal: entre lo ideal, lo real y lo posible. Rev. Latino-Am. Enfermagem. [Internet]. 2011. [Acceso 13 octubre 2018];19(2):08 pantallas., Disponible en: http://www.scielo.br/pdf/rlae/v19n2/es_11.pdf

25. Sánchez-Duque J, Pantoja SA, Pérez J, Patiño AM, Gómez JF. Procedimientos quirúrgicos y unidad de cuidados intensivos correlación epidemiológica en una clínica de tercer nivel de Colombia. Revista Hispanoamericana de Ciencias de la Salud (RHCS). [Internet]. 2016. [Acceso 07 diciembre 2018];3(2):35-40. Disponible en: https:/ /dialnet.unirioja.es/servlet/articulo?codigo $=6230778$

26. Ministerio de s anidad y polític a social. Unidad de cuidados intensivos: estándares y recomendaciones. [Internet]. 2010. [Acceso 23 marzo 2018]. Disponible en: http:/ / www.mscbs.gob.es/ organizacion/sns/planCalidadSNS/docs/UCl.pdf 